\title{
Calcitonin Transport through Skin Using Iontophoresis
}

\author{
Kyungmin Kim and Seaung-youl $\mathrm{Oh}^{\dagger}$
}

College of Pharmacy, Sookmyung Women's University, Seoul 140-742, Korea

(Received December 1, $2010 \cdot$ Revised December 17, 2010 • Accepted December 18, 2010)

\begin{abstract}
The objective of this work is to study transdermal delivery of calcitonin using iontophoresis and to evaluate various factors which affect the transdermal transport. We have studied the effect of polarity, current density, drug concentration, penetration enhancers (isopropyl myristate [IPM] and ethanol) and laser treatment on transdermal flux and the results were compared. We also investigated the iontophoretic flux from microemulsions containing calcitonin together with oleic acid (OA) or IPM. In vitro flux study was performed at $33^{\circ} \mathrm{C}$, using side-by-side diffusion cell and full thickness hairless mouse skin. Anodal delivery at $\mathrm{pH} 3.0$ was much larger than cathodal and passive delivery, due to the positive charge of calcitonin. Cumulative amount delivered (CUM) by cathodal or passive delivery was close to zero for 10 hours. The pretreatment of skin by neat IPM markedly increased the CUM anodically. CUM increased as the current density, drug concentration or the duration of IPM treatment increased. Microemulsion containing IPM or oleic acid was prepared and the phase diagram was constructed. CUM also increased when IPM was incorporated into a microemulsion. OA microemulsion showed similar enhancing effect to IPM microemulsion. The delivery of calcitonin from $70 \%(\mathrm{v} / \mathrm{v})$ ethanol aqueous solution showed a large increase in flux. Laser treatment of skin before flux experiment exhibited about 2 fold increase in total calcitonin amount transported for 12 hours, when compared to that delivered by IPM microemulsion. Based on these results, we have evaluated the possibility of delivering enough amount of calcitonin to reach the therapeutic level. The data suggest that it is highly possible to deliver clinically effective amount of calcitonin using iontophoresis patch with small area $(<10$ $\mathrm{cm}^{2}$ ).
\end{abstract}

Key words - Iontophoresis, Calcitonin, Isopropyl myristate, Oleic acid, Microemulsion, Laser

Calcitonin is a cyclic polypeptide of 32 amino acid and an important role in the regulation of calcium homeostasis, and is known to act as a potent inhibitor of osteoclastic bone resorption (Patel et al., 1993). Due to its short half-life (approximately 15-20 min) after parenteral administration, frequent injection was required to maintain its therapeutic effect (Huwyier et al., 1979). However, injections are inconvenient for outpatient use and frequently lead to compliance problems when self administration is required. Various dosage forms, such as intranasal, transdermal and oral delivery systems have been developed, in order to increase the duration of action with increased bioavailability (Lamprecht et al., 2004; Matsuyama et al., 2006; Nakamura et al., 2001).

Transdermal delivery has many advantages over other dosage forms, such as large application surface area, ease of application and removal, maintaining constant blood concentration and long term delivery (Ramachandran et al., 2000). However, only about 20 drug molecules have been formulated successfully as transdermal products, due to the barrier property of

\footnotetext{
†Corresponding Author:

Tel : +82-2-710-9563, E-mail : syoh@sookmyung.ac.kr DOI : 10.4333/KPS.2011.41.1.009
}

stratum corneum (SC) (Subedi et al., 2010). Hence, it is very important to develop various penetration enhancing methods to enlarge the number of drug molecules to be in the transdermal range. In order to modify the SC and to increase the permeability, various physical and chemical methods have been developed, including chemical penetration enhancers and iontophoresis (Ibrahim et al., 2010; Kigasawa et al., 2010). Iontophoresis has been known over 90 years, since Leduc showed that strychnine sulfate can be delivered to rabbit (Leduc, 1908). However, it has gained new interest in 1980's, because of its potential to deliver peptide drugs, which were becoming available by the advancement of genetic engineering but were very difficult to deliver by conventional methods (Green et al., 1992). Iontophoresis provides a safe, economical, and convenient way to deliver both charged and uncharged drug molecules in a controllable manner. Iontophoresis has been found useful in lidocaine and fentanyl citrate delivery for pain control (Zempsky et al., 1998; Power, 2007) and the monitoring of blood glucose level for diabetes (Potts et al., 2002).

The objective of this work is to study transdermal delivery of calcitonin (M.W. 3431.9) using iontophoresis in combination with various other enhancing methods, and to assess the feasibility of delivering therapeutic amount of calcitonin 
through skin, though the molecular weight of calcitonin is rather large as a candidate for usual transdermal delivery. In order to increase the flux further, we have employed several chemical enhancing methods such as neat IPM treatment, microemulsion formulation using IPM and OA, and ethanol aqueous solution treatment. As a physical invasive flux enhancement method, we also investigated the application of $\mathrm{CO}_{2}$ laser. The combination of the iontophoresis with other permeation enhancing method usually showed increased delivery and synergistic effect. The study of Bhatia et al. (1997) showed that skin pretreatment with chemicals increased the effect of iontophoresis on transdermal transport of luteinizinghormone-releasing-hormone (LHRH). The chemical enhancers used in this work have been used widely in the transdermal formulation. IPM was known to impair the lipid barrier of the skin (Suh et al., 1996). The effects of isopropyl myristate (IPM) as an enhancer for the permeation have been investigated by Suwanpidokkul et al. (Suwanpidokkul et al., 2004). Ethanol/IPM was tested for azidothymidine solubility and permeability across pig skin. Ethanol/IPM (50/50, v/v) demonstrated high azidothymidine flux. IPM also improved iontophoretic permeation (Batheja et al., 2006). Microemulsions have been proposed to offer enhanced drug delivery properties for transdermal transport. It has been reported that microemulsion increased the permeation rate of dexamethasone, suggesting microemulsion-based systems can be a promising formulation for dermal delivery (Chandra et al., 2009). OA has been widely used as transdermal enhancer. OA in propylene glycol (PG) was markedly successful in increasing the permeation rate of 5-FU and estradiol through human skin. Results suggested that OA remained in tissues for longer periods (Goodman et al., 1988). The laser has been suggested for the controlled ablation or removal of the SC in human skin (Jacques et al., 1987) and it is reported that the procedure for $\mathrm{SC}$ ablation by $\mathrm{CO}_{2}$ laser is accompanied by heating (Marcells et al., 2000).

\section{Materials and Methods}

\section{Materials and instruments}

All reagents and chemicals were of the highest commercially obtainable purity. Sodium phosphate monobasic, sodium phosphate dibasic, bezalkonium chloride and HPLC grade tetramethylammonium hydroxide (TMA) were purchased from Sigma-Aldrich Co. (Seoul, Korea). Isopropyl myristate (IPM), oleic acid (OA), HPLC grade acetonitrile and sodium chloride were obtained from Duksan Chem. (Seoul, Korea). Buffer solutions were prepared using distilled water from Nanopure ultrapure water system D11921 (Barnstead, Dubuque, IA, USA). Calcitonin was obtained from Soho-Yiming Pharmaceutical Co., Ltd. (Shanghai, China). The instruments used were as follow: power supply (Model PT 70-10 MDC, Power Tech, Ansan, Korea), incubator (SI-900, Jeio Tech, Ansan, Korea), pH meter (Model 320, Corning, New York, NY, USA), HPLC system (Futecs NS-4000, Daejeon, Korea), deep freezer (Model 925, Forma Scientific Inc., Marjetta, OH, USA), ultrasonicator (Model 5210, Brason, Danbury, CT, USA), laser (CICU-F, Ilooda Co., Suwon, Korea).

\section{Preparation of electrodes}

$\mathrm{Ag} / \mathrm{AgCl}$ electrodes were used for their stability and reversibility. The rod-shaped electrodes were prepared by dipping the tip of an Ag wire (99.9\%, Aldrich, Milwaukee, WI, USA) (1 $\mathrm{mm}$ diameter) into the molten $\mathrm{AgCl}$.

\section{Preparation of the microemulsion formulations}

IPM or OA was selected as an oil component and ethanol was used as co-surfactant in the microemulsion systems. The surfactant, Tween 40 or Tween 80 , was prepared separately. The pseudo-ternary phase diagrams of oil, surfactant/co-surfactant, and water were set up using the water titration method. IPM microemulsion was oil/water type and contained $8.6 \%$ oil, 5.3\% ethanol, $51.6 \%$ buffer and $34.5 \%$ surfactant (Tween 40). OA microemulsion was formulated by the method of Jung et al. (2008) and contained 3\% oil, 33.8\% ethanol, 50\% buffer and $13.2 \%$ surfactant (Tween 80 ).

\section{Drug assay}

To assay calcitonin, a calcitonin EIA kit (Phoenix Pharmaceuticals, Inc., Belmont, CA, USA) was used for very low concentration of calcitonin. Standards, positive controls, and samples were added to anti-calcitonin antibody coated strip wells in triplicate, and were incubated with another biotinylated anti- calcitonin antibody. The plate was washed after the incubation, and prepared streptavidin enzyme conjugate was added to the plate, which was subsequently incubated. The plate was again washed after the incubation, and TMB substrate solution was added. Color was allowed to develop before stopping the reaction with kit Stop solution. Absorbance was measured at $450 \mathrm{~nm}$. Concentration was calculated by interpolation from a standard curve and assay performance was controlled with positive control samples. For higher concentration of calcitonin, high performance liquid chromatograph were employed (Futecs NS-4000). The chromatograph was equipped with an automatic sampler injector (Futecs NS6000 ), a variable wavelength UV detector and a reversed phase 
column (Atlantis ${ }^{\circledR} \mathrm{dc} 183 \mu \mathrm{m}, 4.6 \times 150 \mathrm{~mm}$, Waters, Milford, MA, USA). The column temperature was maintained at $50^{\circ} \mathrm{C}$. The mobile phase was A (1 M TMA-DW-ACN, 20:880:100) 63 : B (1 M TMA-DW-ACN, 8:392:600) 37. Solutions A and $\mathrm{B}$ are adjusted to $\mathrm{pH} 2.5$ with phosphoric acid. The UV detection was at $210 \mathrm{~nm}$, with a flow rate $1 \mathrm{~mL} / \mathrm{min}$.

\section{Skin permeation}

Calcitonin transport experiments were made using full-thickness hairless mouse skin. The skin was excised and frozen immediately after sacrifice of 8 week old mouse (Orient Bio Inc., Seongnam, Korea). It was stored at $-70^{\circ} \mathrm{C}$ and then thawed just before use. A side-by-side diffusion cell (Yuil Science, Busan, Korea) was used. The chamber held a volume of $1 \mathrm{~mL}$ and was magnetically stirred. The area of skin exposed to each chamber was $0.5 \mathrm{~cm}^{2}$. Skin was treated with neat IPM for predetermined time, and washed with buffer solution several times before flux experiment. The donor and receptor chamber were filled with phosphate buffer solution $(\mathrm{pH} 3.0)$ containing calcitonin and phosphate buffer solution ( $\mathrm{pH}$ 7.0), respectively. Microemulsion or ethanol solution was directly placed into the donor solution. Laser treatment was made by the programmed short pulse and the laser wavelength was $10.6 \mu \mathrm{m}$. At a predetermined time interval, sampling was made and an equal volume of $1 \mathrm{~mL}$ phosphate buffered solution at $\mathrm{pH} 7.0$ was added to the receptor chamber. Experiments were conducted at $33^{\circ} \mathrm{C}$ using an incubator (SI-900, Jeio Tech).

\section{Results and Discussions}

\section{Effect of polarity}

The delivery of calcitonin by cathodal and anodal iontophoresis together with passive diffusion is shown in Fig. 1. Passive permeation and cathodal delivery of calcitonin across hairless mouse skin were negligible and no calcitonin could be detected. Anodal delivery showed small amount of calcitonin transported. This is probably due to the positive charge of calcitonin at the $\mathrm{pH}$ studied ( $\mathrm{pH} 3.0)$. Because the amount of calcitonin transported was very insignificant, we used calcitonin EIA kit (Phoenix Pharmaceuticals, Inc.) for the assay.

\section{Effect of IPM pretreatment}

Fig. 2 shows the effect of neat IPM pretreatment on CUM. When skin was not treated, flux of calcitonin was not detected at all. When skin was treated for 3 hours before flux experiment, marked increase in CUM for 12 hours was observed, when compared to that obtained without IPM treatment $(p<0.05)$. As the duration of IPM pretreatment increased to 12

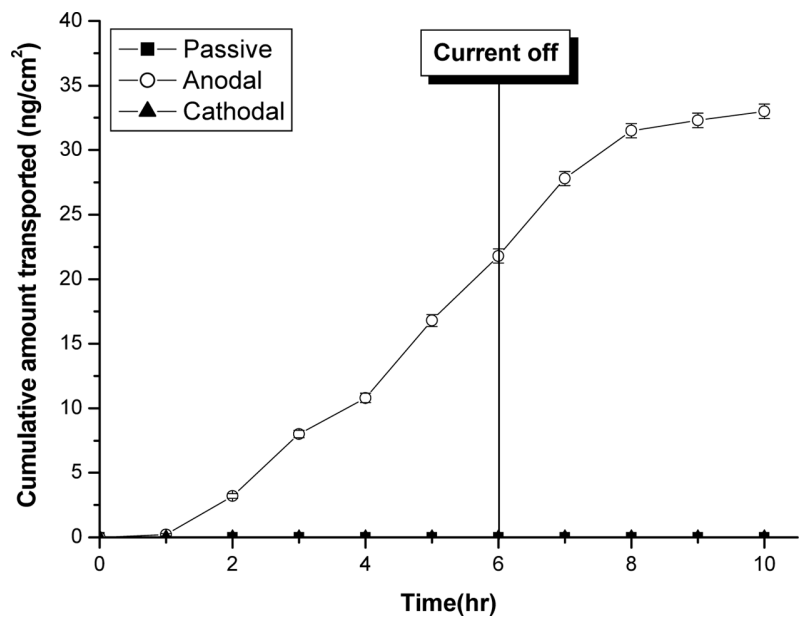

Figure 1. Cumulative amount of calcitonin transported from $1 \mathrm{mg} /$ $\mathrm{mL}$ solution across hairless mouse skin in vitro. Current of $0.3 \mathrm{~mA} /$ $\mathrm{cm}^{2}$ was applied for 6 hours. Each data point represents the mean $( \pm \mathrm{SD})$ of 3 experiments (at $33^{\circ} \mathrm{C}$ incubator).

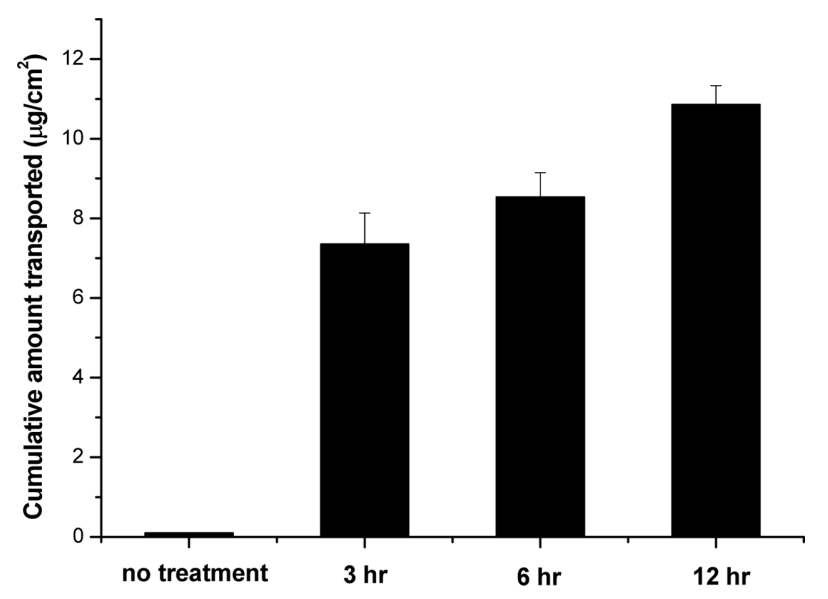

Figure 2. The effect of IPM pretreatment time on the cumulative amount of calcitonin transported from donor solution containing $2 \mathrm{mg} / \mathrm{mL}$ of calcitonin. Each data point represents the mean $( \pm \mathrm{SD})$ of 3 experiments (at $33^{\circ} \mathrm{C}$ incubator).

hours, the delivery of calcitonin increased further $(\mathrm{p}<0.05)$. The CUM for 3, 6 and 12 hour pretreatment was 7.4, 8.5 and $10.9 \mu \mathrm{g} / \mathrm{cm}^{2}$. The effectiveness and mode of action of IPM as an enhancer for the permeation have been investigated by Suh et al. (1996). By observing similar permeation of naproxen through intact and delipidized skin after IPM treatment, they concluded that the lipid barrier of the skin was largely impaired by IPM. They showed that neat application of IPM to skin is more effective than the gel formulation. Pillai et al. (2004) investigated the effect of pre-treatment with IPM on insulin iontophoresis. They showed that IPM was able to produce a synergistic enhancement with iontophoresis and FT-IR 


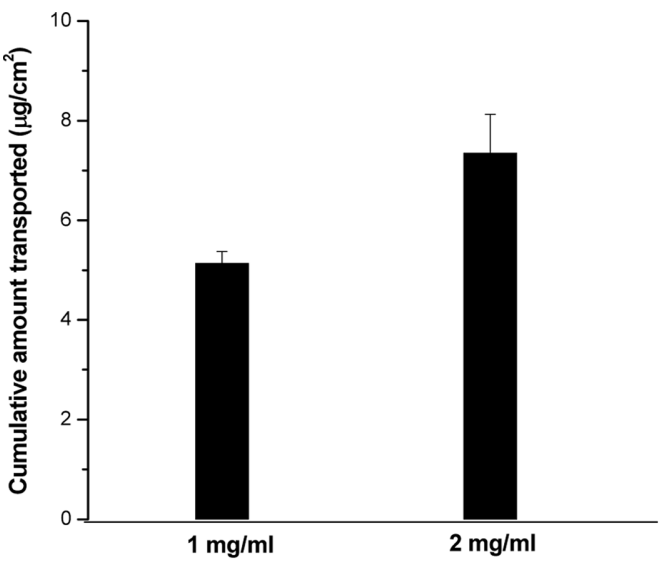

(a)

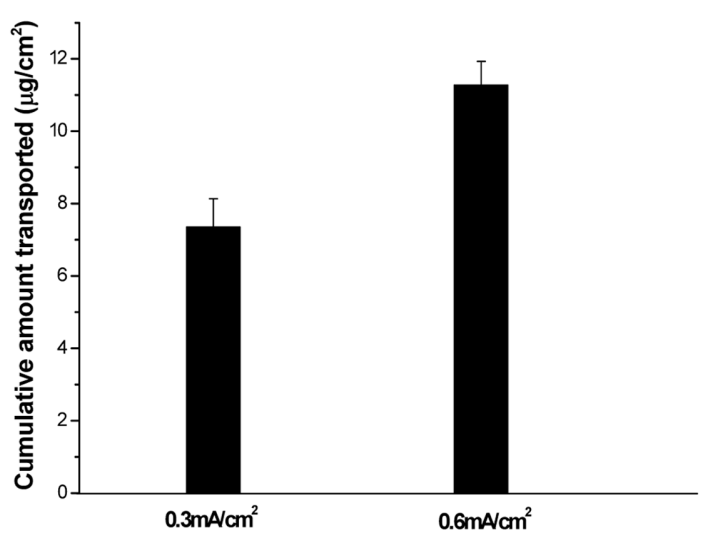

(b)

Figure 3. (a) The effect of drug concentration on the cumulative amount of calcitonin transported by anodal delivery after IPM pretreatment for 3 hours. The current density was $0.3 \mathrm{~mA} / \mathrm{cm}^{2}$. (b) The effect of anodal current density on the cumulative amount of calcitonin transported from donor solution containing $2 \mathrm{mg} / \mathrm{mL}$ of calcitonin. Skin was treated with IPM for 3 hours before current application. Each data point represents the mean $( \pm \mathrm{SD})$ of 3 experiments (at $33^{\circ} \mathrm{C}$ incubator).

studies revealed that IPM caused increase in lipid fluidity of the SC lipid. This increase in fluidity disrupts the lipid orderness in SC, and lowers the barrier property of the stratum corneum, causing the decrease in resistance and increase in capacitance. Fig. 3(a) shows the influence of calcitonin concentration on the iontophoretic flux after IPM pretreatment for 3 hours. When calcitonin concentration was $2 \mathrm{mg} / \mathrm{mL}$, the CUM for 12 hours was $7.4 \mu \mathrm{g} / \mathrm{cm}^{2}$, and this value was about 1.4 fold larger than the CUM obtained for $1 \mathrm{mg} / \mathrm{mL}(5.2 \mu \mathrm{g} /$ $\mathrm{cm}^{2}$ ). The effect of current density on the iontophoretic flux of calcitonin is shown in Fig. 3(b). Increase in current density from 0.3 to $0.6 \mathrm{~mA} / \mathrm{cm}^{2}$ resulted in an increase in the CUM $(p<0.05)$. When the current density was $0.6 \mathrm{~mA} / \mathrm{cm}^{2}$, the CUM was $11.3 \mu \mathrm{g} / \mathrm{cm}^{2}$. This value is about 1.5 fold larger than that observed when the current density was $0.3 \mathrm{~mA} / \mathrm{cm}^{2}(7.4 \mu \mathrm{g} /$ $\mathrm{cm}^{2}$ ).

According to Nernst-Plank, flux by iontophoresis can be described by the following equation (Heit et al., 1997).

$$
\begin{aligned}
& J_{t}=-D_{i}\left(\frac{d C_{i}}{d X}+\frac{C_{i} Z_{i} F}{R T} \frac{d V}{d X}\right)+V C_{i} \\
& J_{t}=J_{\text {diffusion }}+J_{\text {electrorepulsion }}+J_{\text {electroosmosis }}
\end{aligned}
$$

Where $J$ is flux, $F$ is Faraday constant, $D$ is diffusion coefficient, $C$ is concentration of ioninc drug molecule, $Z$ is the charge of the ion, $T$ is absolute temperature, $V$ is the potential applied to the skin and $\mathrm{R}$ is the Boltzmann constant. This equation indicates that total flux by iontophoresis is the sum of passive diffusional flux by concentration gradient, flux by electrorepulsion by potential gradient and the flux by electro osmotic flow due to the permselectivity of skin. The results in Fig. 23 indicate that electrorepulsion is the main driving force for the transport, because the $\mathrm{pH}$ of solution in contact with stratum corneum is $\mathrm{pH}$ 3.0. At this $\mathrm{pH}$, the permselectivity of skin is probably reversed and the direction of ectroosmosis is cathode to anode. Thus the flux by electro-repulsion is hindered by the electroosmotic flow. This impeding effect of flux is probably larger for higher current, because the magnitude of electroosmotic flow increases as the current density increases..

\section{Iontophoresis using microemulsions}

Microemulsions are thermodynamically stable systems of water, oil, surfactant, and co-surfactant and are known to enhance the bioavailability of drugs via topical and systemic routes. Microemulsions have been considered as topical, transdermal (Thevenin et al., 1996), parenteral and vaginal drug delivery systems based on their favorable solubilization and transport enhancement properties. Three main mechanisms had been proposed to explain the advantages of microemulsion for the transdermal delivery of drugs (Delgado-Charro et al., 1997). First, a large amount of drug can be included in the formulation due to the high solubilization power. Second, an increase in the transdermal flux can be expected in that the thermodynamic activity of the drug in the microemulsion can be modified to favour partitioning into the stratum corneum. Third, the surfactants in the microemulsion may reduce the diffusional barrier of the stratum corneum. Ingredients of microemulsion, acting as permeation enhancers, might destroy the structure of stratum corneum and increase the flux of drug via skin (Kreilgaard, 2002). Combination of iontophoresis and 
microemulsion formulation to facilitate skin penetration of lidocaine was investigated (Sintov et al., 2006). It was shown in vitro that by combining microemulsion application with iontophoresis, a significantly increased flux was obtained compared with a combination of aqueous drug solution with the same iontophoresis protocol.

In this work, we have tried to prepare two microemulsion systems (IPM \&OA microemulsion), and their flux enhancing ability under iontophoresis was investigated. The pseudo-ternary phase diagram of the IPM microemulsion system is shown in Fig. 4. IPM microemulsion was oil/water type and the composition of microemulsion used in this experiment contained $8.6 \%$ oil, $5.3 \%$ ethanol, $51.6 \%$ buffer and $34.5 \%$ surfactant (Tween 40). Fig. 5 shows the CUM of calcitonin from IPM microemulsion containing $1 \mathrm{mg} / \mathrm{mL}$ calcitonin for 12 hours. Current was applied for 9 hours and after that passive

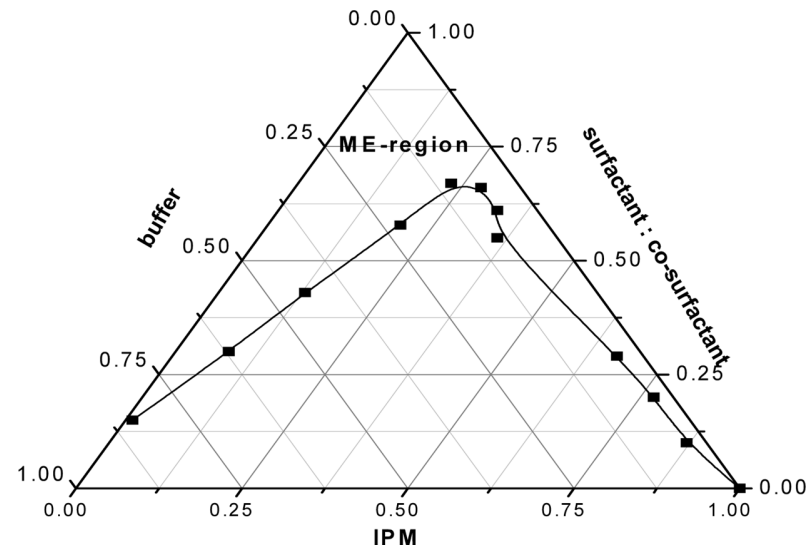

Figure 4. Pseudo-ternary phase diagrams of microemulsion composed of IPM, surfactant (Tween 40), co-surfactant (ethanol) and phosphate buffer ( $\mathrm{Km} \mathrm{0.55).}$

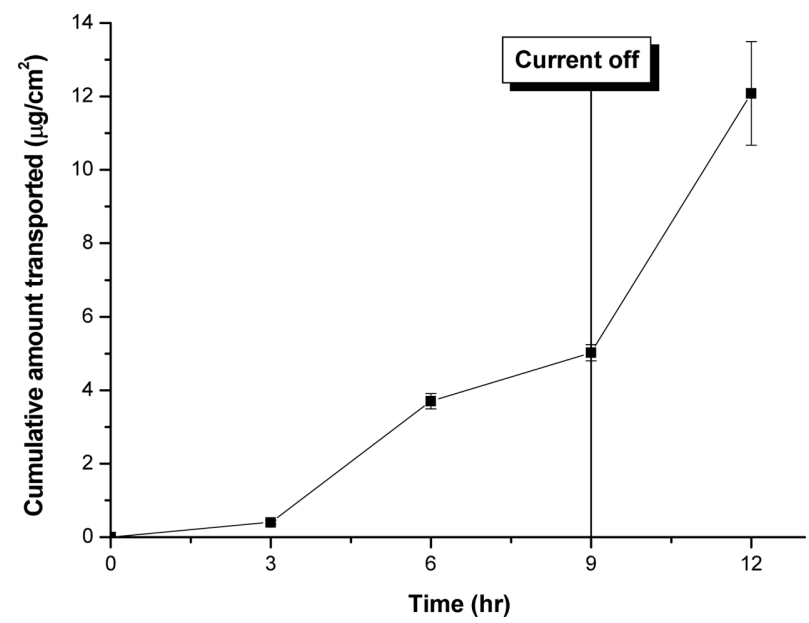

Figure 5. Cumulative amount transported of calcitonin from IPM microemulsion across hairless mouse skin in vitro. Each data point represents the mean $( \pm \mathrm{SD})$ of 3 experiments (at $33^{\circ} \mathrm{C}$ incubator). flux was measured for 3 hours. A remarkable increase in the permeation was found. After 9 hours of $0.3 \mathrm{~mA} / \mathrm{cm}^{2}$ current application, CUM was $5.0 \mu \mathrm{g} / \mathrm{cm}^{2}$, and the CUM at 12 hour was $12.1 \mu \mathrm{g} / \mathrm{cm}^{2}$. Rapid increase in flux seems to be related to the disappearance of electroosmotic flow and the increased passive flux. More flux study without current from the 0 hour might provide some more insight into the role of electroosmosis. The CUM at 12 hour was $12.1 \mu \mathrm{g} / \mathrm{cm}^{2}$ and, hence, a $5 \mathrm{~cm}^{2}$ patch containing calcitonin in an IPM microemulsion system would provide $60 \mu \mathrm{g}$ for 12 hours. Because the daily dosage of calcitonin is about 10-100 $\mu \mathrm{g}$ /day (Reginster, 1993; Tascioglu et el., 2005)), it seems reasonable to assume that therapeutically effective amount of calcitonin can be delivered through skin using the simultaneous application of IPM microemulsion and iontotophoresis system.

The pseudo-ternary phase diagram of the OA microemulsion system is shown in Fig. 6. The composition of microemulsion used in this experiment contained 3\% oil, 33.8\% ethanol, $50 \%$ buffer and $13.2 \%$ surfactant (Tween 80 ). Fig. 7 shows the CUM of calcitonin from OA microemulsion containing $1 \mathrm{mg} /$ $\mathrm{mL}$ calcitonin for 12 hours. Current was applied for 9 hours and after that passive flux was measured for 3 hours. After 0.3 $\mathrm{mA} / \mathrm{cm}^{2}$ current application, similar to IPM microemulsion, a remarkable increase in the amount of penetration was found. After 9 hours, CUM was $4.3 \mu \mathrm{g} / \mathrm{cm}^{2}$, and the CUM at 12 hour was $9.6 \mu \mathrm{g} / \mathrm{cm}^{2}$. At higher current, slight increase in CUM was observed during current application. When the current density was $0.5 \mathrm{~mA} / \mathrm{cm}^{2}$, the CUM increased about 1.5 fold $(6.63 \mu \mathrm{g} /$ $\mathrm{cm}^{2}$ ) of the CUM at $0.3 \mathrm{~mA} / \mathrm{cm}^{2}$. The use of iontophoresis and OA on permeation of arginine vasopressin through rat skin was investigated. SD rat skin was pretreated with OA for 2 hours and iontophoresis in vitro, separately or together. The

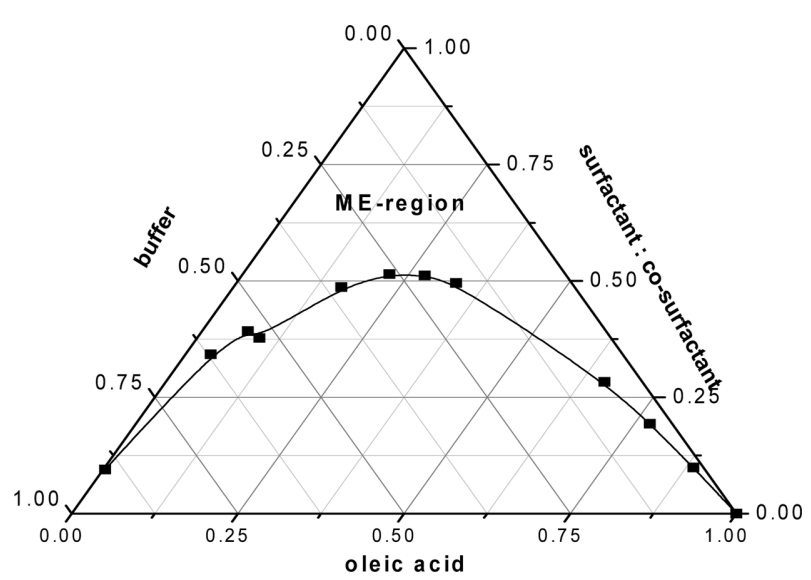

Figure 6. Pseudo-ternary phase diagram of microemulsion composed of OA, surfactant (Tween 80), co-surfactant (ethanol) and phosphate buffer (Km 0.55) (revised from Jung, et al., 2008). 


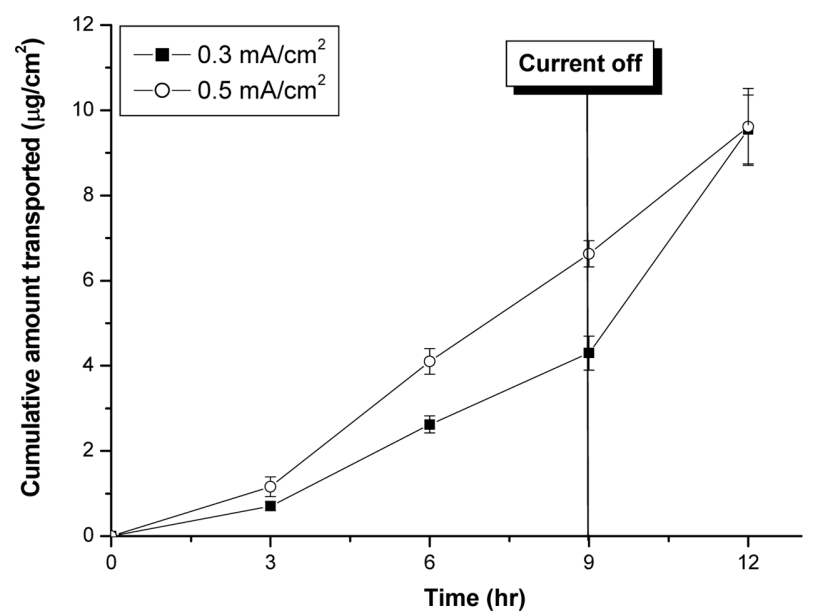

Figure 7. Cumulative amount transported of calcitonin from OA microemulsion across hairless mouse skin in vitro. Anodal current 0.3 or $0.5 \mathrm{~mA} / \mathrm{cm}^{2}$ was applied for 9 hours. Each data point represents the mean $( \pm \mathrm{SD})$ of 3 experiments (at $33^{\circ} \mathrm{C}$ incubator).

results indicate that $\mathrm{OA}$ increased the flux of arginine vasopressin in comparison to control. OA in combination with iontophoresis significantly increased the permeation of arginine vasopressin (Nair et al., 2003).

$\mathrm{OA}$ is an unsaturated fatty acid with 18 carbon atoms. It has been known to be effective enhancers for polar and moderately polar drug molecules. Based on the differential scanning calorimetry (DSC) and fourier transform infrared spectroscopy (FTIR) studies, it has been postulated that OA exists in a separate phase as liquid in the $\mathrm{SC}$ and forms a number of permeable defects at the liquid-solid (SC lipids) (Ongpipattanakul et al., 1991; Francoeur et al., 1990). Loftsson et al. (1989) showed that mixtures of PG with OA exhibit synergism in the permeability of drugs. One possible explanation for this synergistic effect is the facilitated incorporation of OA into the SC lipid alkyl domain by the interaction of PG at the polar head group region. Ultrastructural observations demonstrated both marked disorganization of the intercellular lipid lamellae, as well as the presence of distended lacunae within the stratum corneum in OA/propylene glycol skin (Jiang et al., 2000).

\section{Effect of ethanol}

Ethanol is widely used as a penetration enhancer of drugs in many pharmaceutical products, such as cream, ointments and gels, as well as transdermal controlled release products. It is also commonly used in topical and transdermal products because it is a good solvent for many organic compounds The effect of $70 \%$ ethanol aqueous solution containing $1 \mathrm{mg} / \mathrm{mL}$ of calcitonin on delivery was investigated and the results are shown in Fig. 8. Current was applied for 9 hours and after that passive transport was measured for 3 hours. Similar to micro-

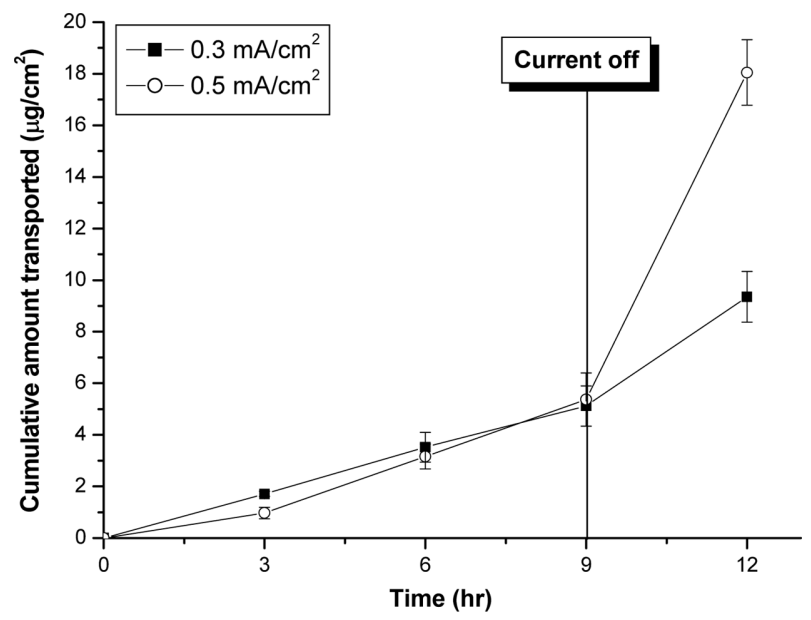

Figure 8. Cumulative amount of calcitonin transported from $70 \%$ ethanol solution (ethanol:buffer $=7: 3$ ) containing $1 \mathrm{mg}$ / $\mathrm{mL}$ of calcitonin. Anodal current was applied for 9 hours $(\mathrm{n}=3)$. $\mathbf{\square}: 0.3 \mathrm{~mA} / \mathrm{cm}^{2}, \circ: 0.5 \mathrm{~mA} / \mathrm{cm}^{2}$.

emulsions, a remarkable increase in permeation was found. After 9 hours of $0.3 \mathrm{~mA} / \mathrm{cm}^{2}$ current application, CUM was $5.1 \mu \mathrm{g} / \mathrm{cm}^{2}$. After the current off, detectable permeation of calcitonin through the stratum corneum occurred, and the CUM at 12 hour increased to $9.4 \mu \mathrm{g} / \mathrm{cm}^{2}$. It seems that the passive permeability of skin is increased by the application of current and the ethanol. At higher current $\left(0.5 \mathrm{~mA} / \mathrm{cm}^{2}\right), \mathrm{CUM}$ at 12 hours increased further to $18.1 \mu \mathrm{g} / \mathrm{cm}^{2}$, which is about 1.9 fold larger than that of $0.3 \mathrm{~mA} / \mathrm{cm}^{2}$. The sharp increase in cumulative amount transported after current off for $0.5 \mathrm{~mA} / \mathrm{cm}^{2}$ is not clear, One possible explanation is higher increase in passive permeability by higher current density. Though the mechanism of action of ethanol for enhancing effect is not known clearly, it seems that lipid extraction and osmotic swelling are the main reason for the enhancement effect (Ghanem et al., 1987; Bommannan et al., 1991). Sinha et al. (2000) showed that ethanol interacts with the proteins in stratum corneum and increases the number of free sulfhydryl groups of keratin.

\section{Effect of laser pretreatment}

Lasers are physical devices that have been used for medical diagnosis and therapeutic purposes. This method involves direct and controlled exposure of a laser beam to the skin which results in the ablation of the stratum corneum without significantly damaging the underlying epidermis. Removal of the stratum corneum using this method has been shown to enhance the delivery of lipophilic and hydrophilic drugs (Lee et al., 2002). Both SC ablation and a thermal effect may contribute to the effect of the $\mathrm{CO}_{2}$ laser on skin structure. Fig. 9 shows confocal laser scanning microscopy image of the penetration and distribution of calcein after laser treatment. Skins 
(a) Control $(120 \mu \mathrm{m})$

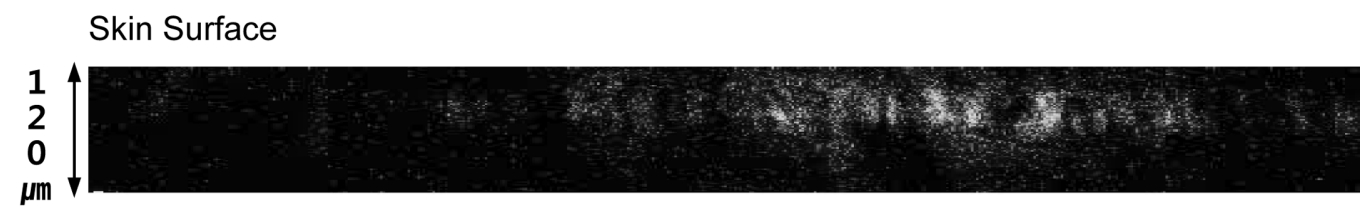

(b) $55 \mathrm{~mJ} / \mathrm{cm}^{2}$ laser $(300 \mu \mathrm{m})$

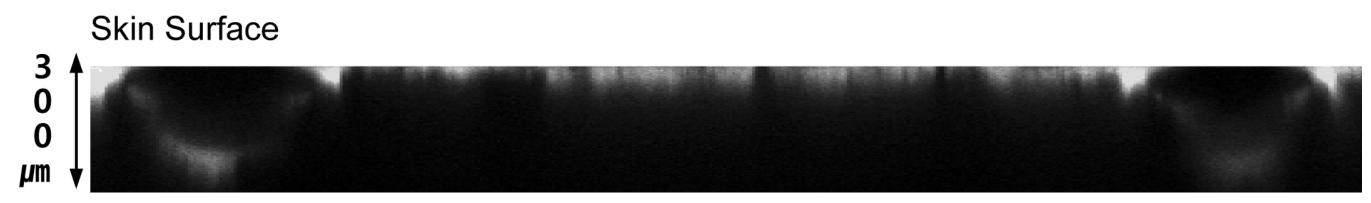

(c) Z-series parallel view

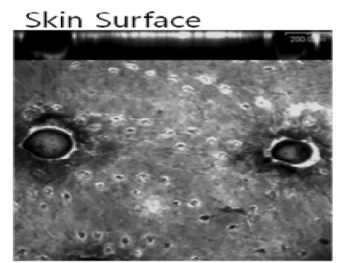

Figure 9. The cross-sectional view (XZ-section; a and b) and Z-series parallel view (c) of calcein penetrated into the skin (100 magnifications).

were treated with laser under the following parameters: wavelength $10.6 \mathrm{im}$; output energy $55 \mathrm{~mJ} / \mathrm{cm}^{2}$. After 1 hour passive penetration, the hairless mouse skin was imaged from the surface of stratum corneum. We acquired a series of optical sections, referred to as a $Z$-series, taken at successive focal planes along the $\mathrm{Z}$ axis from $\mathrm{Z}=0 \mu \mathrm{m}-\mathrm{XY}$-plane to $\mathrm{Z}=300 \mu \mathrm{m}-\mathrm{XY}$ plane. The cross-sectional view (XZ-section) provides information about depth from a specific surface. For the laser nontreated skin (control), only a small amount of calcein penetrated into the top surface of skin. However, for laser treated skin, calcein penetrated deeply into the demis layer, where the laser was irradiated (leftmost and rightmost of Fig. 9(b)). Zseries parallel view.of the laser treated skin is shown in Fig. 9(c). The pore created by the laser treatment can be seen clearly.

Fig. 10 shows the effect of current density on CUM of cacitonin after laser treatment $\left(55 \mathrm{~mJ} / \mathrm{cm}^{2}\right)$. Current was applied for 9 hours and, after that passive transport was measured for 3 more hours. Remarkable increase in permeation was observed at both current densities by the laser treatment. After 9 hours of $0.3 \mathrm{~mA} / \mathrm{cm}^{2}$ current application, CUM was $13.0 \mu \mathrm{g} /$ $\mathrm{cm}^{2}$. After the current off, passive permeation of calcitonin through the stratum corneum was strong, and the CUM at 12 hour increased to $20.3 \mu \mathrm{g} / \mathrm{cm}^{2}$. When the current density was increased to $0.5 \mathrm{~mA} / \mathrm{cm}^{2}$, CUM showed higher value than that

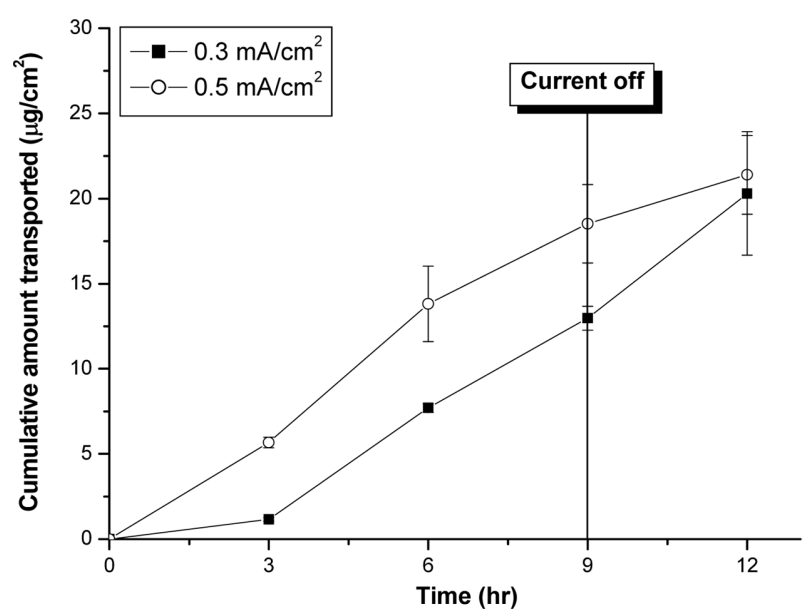

Figure 10. Cumulative amount of calcitonin transported from $1 \mathrm{mg} /$ $\mathrm{mL} \mathrm{pH} 3.0$ buffer solution across hairless mouse skin in vitro after $30 \mathrm{~mJ} / \mathrm{cm}^{2}$ laser application. Anodal Current was applied for 9 hours. Each data point represents the mean $( \pm \mathrm{SD})$ of 3 experiments (at $33^{\circ} \mathrm{C}$ incubator). $0.3 \mathrm{~mA} / \mathrm{cm}^{2}, 0: 0.5 \mathrm{~mA} / \mathrm{cm}^{2}$.

for $0.3 \mathrm{~mA} / \mathrm{cm}^{2}$. CUM was $18.5 \mu \mathrm{g} / \mathrm{cm}^{2}$ and $21.4 \mu \mathrm{g} / \mathrm{cm}^{2}$ after 9 and 12 hours, respectively. Fig. 11 shows calcitonin flux after application of 30 and $50 \mathrm{~mJ} / \mathrm{cm}^{2}$ laser. The CUM after 12 hours was $20.3 \mu \mathrm{g} / \mathrm{cm}^{2}$ for $30 \mathrm{~mJ} / \mathrm{cm}^{2}$ laser treatment. It was $22.8 \mu \mathrm{g} / \mathrm{cm}^{2}$ with $55 \mathrm{~mJ} / \mathrm{cm}^{2}$. These values are about 2 fold larger in total calcitonin amount transported for 12 hours, when compared to that delivered by IPM microemulsion. These flux 


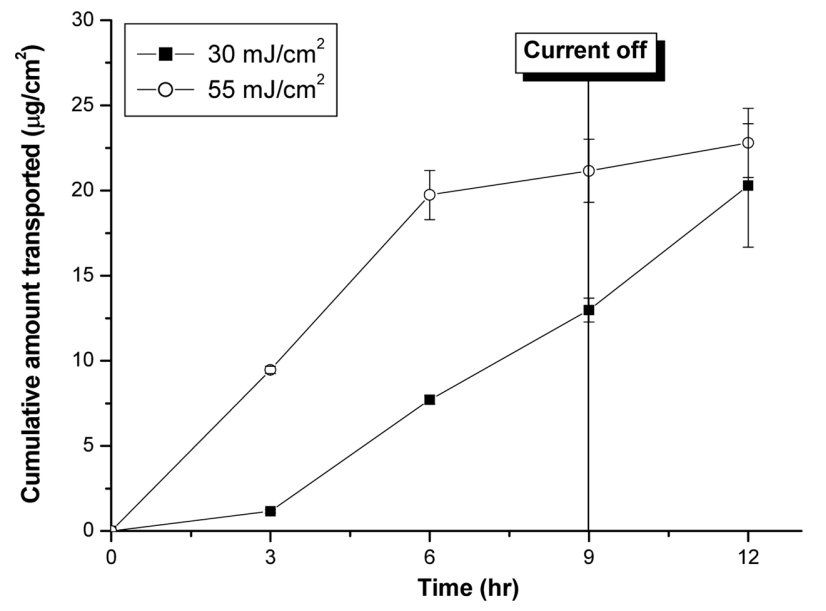

Figure 11. Cumulative amount of calcitonin transported from $1 \mathrm{mg} /$ $\mathrm{mL} \mathrm{pH} 3.0$ buffer solution across hairless mouse skin in vitro after two power levels of laser were applied. Anodal Current was applied for 9 hours. The current density was $0.3 \mathrm{~mA} / \mathrm{cm}^{2}$. Each data point represents the mean $( \pm \mathrm{SD})$ of 3 experiments (at $33 \mathrm{C}$ incubator). : $30 \mathrm{~mJ} / \mathrm{cm}^{2}, \bigcirc: 55 \mathrm{~mJ} / \mathrm{cm}^{2}$.

results, together with the data from non-treated skin, suggest that pores made by the laser treatment are the main route for iontophoretic transport of calcitonin. Based on these results, we have evaluated the possibility of delivering enough amount of calcitonin to reach the therapeutic level. The maximum cumulative amount of calcitonin transported for 12 hours was $22.8 \mu \mathrm{g} / \mathrm{cm}^{2}$ after the application of $55 \mathrm{~mJ} / \mathrm{cm}^{2}$ laser. If the patch size is $5 \mathrm{~cm}^{2}$, then we can deliver $114 \mu \mathrm{g}$ for 12 hours. Because the daily dosage of calcitonin is $10-100 \mu \mathrm{g}$, it seems possible to deliver clinically effective amount of calcitonin using iontophoresis

\section{Conclusions}

In this work, we have investigated the transdermal delivery of calcitonin using iontophoresis. Calcitonin is positively charged at $\mathrm{pH} 3.0$, and anodal delivery was much larger than cathodal or passive delivery. We have studied the effect of the skin penetration enhancer (IPM and ethanol), microemulsions (OA and IPM) and laser treatment. The pretreatment of skin by neat IPM increased anodal flux markedly. Flux was proportional to the increase of drug concentration and current density after IPM pretreatment. The delivery of calcitonin increased remarkably by microemulsion of IPM and OA, 70\% ethanol and laser treatment. Based on these results, we have evaluated the possibility of delivering enough amount of calcitonin to reach the therapeutic level. The data suggest that it is highly possible to deliver clinically effective amount of calcitonin using iontophoresis patch with small area $\left(<10 \mathrm{~cm}^{2}\right)$, though the reversibility of skin after these enhancer treatment still remains to be evaluated.

\section{Acknowledgement}

This Research was supported by a grant of the Ministry of Knowledge Economy, Republic of Korea (10031157).

\section{References}

Bhatia, K.S., Gao, S., Singh. J., 1997. Effect of penetration enhancers and iontophoresis on the FT-IR spectroscopy and LHRH permeability. J. Control. Rel. 47, 81-89.

Bommannan, D., Potts, R.O., Guy, R.H., 1991. Examination of the effect of ethanol on human stratum corneum using infrared spectroscopy. J. Control. Rel. 16, 299-304.

Chandra, A., Sharma, P.K., Irchhiaya, R., 2009. Microemulsionbased hydrogel formulation for transdermal delivery of dexamethasone. Asian. J. Pharmaceutics. 3(1), 30-36.

Delgado-Charro, M.B., Iglesias-Vilas, G., Blanco-Méndez, J., López-Quintela, M.A., Marty, J.P., Guy, R.H., 1997. Delivery of a hydrophilic solute through the skin from novel microemulsion systems. Eur. J. Pharm. Biopharm. 43, 37-42.

Francoeur, M.L., Golden, G.M., Potts, R.O., 1990. Oleic acid: Its effects on stratum corneum in relation to (trans)dermal drug delivery. Pharm. Res. 7, 621- 627.

Ghanem, A.H., Mahmoud, H., Higuchi, W.I., Rohr, U.D., Borsadia, S., Liu, P., Fox, J., Good, W., 1987. The effects of ethanol on the transport of â-estradiol and other permeants in hairless mouse skin II. A New quantitative approach. J. Control. Rel. 6, 75-83.

Goodman M., Barry, B.W., 1988. Action of penetration enhancers on human skin as assessed by the permeation of model drugs 5-fluorouracil and estradiol. I. Infinite dose technique. J. Invest. Dermatol. 91, 323-327.

Green, P.G., Hinz, R.S., Kim, A., Cullander C., Yamane G., Szoka Jr., F.C., Guy, R.H., 1992. Transdermal iontophoresis of amino acids and peptides in vitro, J. Control. Rel. 21(1-3), 187-190.

Heit, M.C., Riviere, J.E., 1997. Electrically-Assisted transdermal drug delivery. Pharm. Res. 14(6), 687-761.

Huwyler, R., Born, W., Ohanhaus, E.E., Fischer, J.A., 1979 Plasma kinetics and urinary excretion of exogenous human and salmon calcitonin in man. Am. J. Physiol. 236, E15-E19

Ibrahim, S.A., Li, S.K., 2010. Chemical enhancer solubility in human stratum corneum lipids and enhancer mechanism of action on stratum corneum lipid domain. Int. J. Pharm. 383, 89-98.

Jacques, S.L., McAuliffe, D.J., Irvin, B.S., Blank, I.H., Parrish, J.A., 1987. Controlled removal of human stratum corneum by pulsed laser. J. Invest. Dermatol. 88, 88-93.

Jiang, S.J., Hwang, S.M., Choi, E.H., Elias, P.M., Ahn, S.K., Lee, S.H., 2000. Structural and functional effects of oleic acid and iontophoresis on hairless mouse stratum corneum. J. Invest. 
Dermatol. 114, 64-70.

Jung, S.A., Gwak, H.S., Chun, I.K., Oh, S.Y., 2008. Iontophoretic Delivery of Levodopa: Permeation Enhancement by Oleic Acid Microemulsion and Ethanol. J. Kor. Pharm. Sci. 38(6), 373-380.

Kigasawa, K., Kajimoto, K., Hama, S., Saito, A., Kanamura, K., Kogure, K., 2010. Noninvasive delivery of siRNA into the epidermis by iontophoresis using an atopic dermatitis-like model rat. Int. J. Pharm. 383, 157-160.

Kreilgaard, M., 2002. Influence of microemulsions on cutaneous drug delivery. Adv. Drug Deliv. Rev. 54, S77-S98.

Lamprecht A, Yamamoto H., Takeuchi H., Kawashima Y., 2004. $\mathrm{pH}$-sensitive microsphere delivery increases oral bioavailability of calcitonin. J. Control. Rel. 98(1), 1-9.

Leduc, S., 1908. Electric ions and their use in medicine. Rebman Ltd, London.

Lee, W.R., Shen, S.C., Wang, K.H., Hu, C.H., Fang, J.Y., 2002. The effect of Laser treatment on skin to enhance and control transdermal delivery of 5-flurouracil. J.Pharm. Sci. 91 (7), 1613-26.

Loftsson, T., Gildersleeve, N., Bodor, N., 1989. Effect of choline esters and oleic acid on the penetration of acyclovir, estradiol, hydrocortisone, nitroglycerin, retinoic acid and trifluorothymidine across hairless mouse skin in vitro. Acta. Pharm. Nord. 1, 279-286

Marcells, G.N., Ellis, D.A.F., 2000. Laser facial skin resurfacing: discussion on erbium:YAG and CO2 lasers. J. Otolaryngol. 29 , 78-82.

Matsuyama, T., Morita, T., Horikiri, Y., Yamahara, H., Yoshino, H., 2006. Improved nasal absorption of salmon calcitonin by powdery formulation with N-acetyl-L-cysteine as a mucolytic agent. J. Control. Rel. 115(2), 183-188.

Nair, V.B., Panchagnula, R., 2003. Effect of iontophoresis and fatty acids on permeation of arginine vasopressin through rat skin Pharmacol. Res. 47(6), 563-569.

Nakamura K., Kazuya K., Mori K., Higo N., Sato S., Yamamoto K., 2001. Transdermal administration of salmon calcitonin by pulse depolarization-iontophoresis in rats. Int. J. Pharm. 218(12), 93-102.

Ongpipattanakul, B., Burnette, R.R., Potts, R.O., Francoeur, M.L., 1991. Evidence that oleic acid exists in a separate phase within stratum corneum lipids. Pharm. Res. 8(3), 350-354.

Patel, S., Lyons, A.R., Hosking, D.J., 1993. Drugs used in the treatment of metabolic bone diseases. Drugs. 46, 594-617

Pillai, O., Nair, V., Panchagnula, R., 2004. Transdermal ionto- phoresis of insulin: IV. Influence of chemical enhancers. Int. J. Pharm. 269, 109-120.

Potts, R.O., Tamada, J.A., Tierny M.J., 2002. Glucose monitoring by reverse iontophoresis. Diabetes. Metab. Res. rev. 18(S1), S49-S63.

Power, I., 2007. Fentanyl $\mathrm{HCl}$ iontophoretic transdermal system (ITS): clinical application of iontophoretic technology in the management of acute postoperative pain. Br. J. Anaesth. 98(1), 4-11.

Batheja, P., Thakur, R., Michniak, B., 2006. Transdermal iontophoresis. Expert Opin. Drug Deliv. 3(1), 127-138.

Ramachandran, C., Fleisher, D., 2000. Transdermal delivery of drugs for the treatment of bone diseases. Adv. Drug Deliver. Rev. 42, 197-223.

Reginster, J.Y., 1993. Calcitonin for prevention and treatment of osteoporosis. Am. J. Med. 95(5A), 44S-47S.

Sinha V.R., Maninder P.K., 2000. Permeation Enhancers for Transdermal Drug Delivery. Drug Dev. Ind. Pharm. 26(11), 11311140.

Sintov, A.C., Brandys-Sitton, R., 2006. Facilitated skin penetration of lidocaine: combination of a short-term iontophoresis and microemulsion formulation. Int. J. Pharm. 316(1-2), 58-67.

Subedi, R.K., Oh, S.Y., Chun, M.K., Choi, H.K., 2010. Recent advances in transdermal drug delivery. Arch. Pharm. Res. 33, 339-351.

Suh, H., Jun, H.W., 1996. Effectiveness and mode of action of isopropyl myristate as a permeation enhancer for naproxen through shed snake skin. J. Pharm. Pharmacol. 48, 812-816.

Suwanpidokkul, N., Thongnopnua, P., Umprayn, K., 2004. Transdermal delivery of zidovudine (AZT): the effects of vehicles, enhancers, and polymer membranes on permeation across cadaver pig skin. AAPS PharmSciTech. 5(3), 48.

Tascioglu, F., Colak, O., Armagan, O., Alatas, O., Oner, C., 2005. The treatment of osteoporosis in patients with rheumatoid arthritis receiving glucocorticoids: a comparison of alendronate and intranasal salmon calcitonin. Rheumatol. Int. 26(1), 21-9.

Thevenin, M.A., Grossiord, J.L., Poelman, M.C., 1996. Sucrose esters/co-surfactant ME systems for transdermal delivery: assessment of bicontinuous structures. Int. J. Pharm. 137, 177186.

Zempsky W.T., Anand K.J.S., Sullivan, K.M., Fraser, D., Cucina, K., 1998. Lidocaine iontophoresis for topical anesthesia before intravenous line placement in children. J. Pediatrics. 132(6), 1061-1063. 